\title{
Does the Implementation of an Emergency Nursing Framework (HIRAID) Reduce patient Deterioration? A Multi-Centre Quasi-Experimental Study
}

Kate Curtis ( $\square$ kate.curtis@sydney.edu.au )

The University of Sydney

Belinda Munroe

The University of Sydney

Margaret Fry

University of Technology Sydney

Julie Considine

Deakin University School of Nursing and Midwifery

Erin Tuala

Wollongong Hospital

Michael Watts

Wollongong Hospital

Hatem Alkhouri

NSW Agency for Clinical Innovation: New South Wales Agency for Clinical Innovation

Tiana Elphick

The University of Sydney

Kate Ruperto

Wollongong Hospital

Jacquie Barrass

Wollongong Hospital

Sharyn Balzer

Shoalhaven District Memorial Hospital

Bronwynne Chisholm

Illawarra Shoalhaven Local Health District

Connie Van

The University of Sydney

Ramon Z Shaban

The University of Sydney

Research 
Keywords: Adverse events, Emergency, Nursing practice, Assessment, Clinical effectiveness, Patient safety

Posted Date: October 16th, 2020

DOI: https://doi.org/10.21203/rs.3.rs-91144/v1

License: (1) This work is licensed under a Creative Commons Attribution 4.0 International License. Read Full License 


\section{Abstract}

Background: Recognition and response of deteriorating patients is fundamental to safe, high quality healthcare. Failure to recognise and respond to deteriorating patients is associated with serious adverse events, both during and after emergency department care. This study aims to determine if the implementation of HIRAID (History, Identify Red flags, Assessment, Interventions, Diagnostics, communication and reassessment), an emergency nursing framework, improves patient safety.

Methods: This quasi-experimental cohort study was conducted between November 2017 and February 2019 in two regional hospitals in [Anonymised], Australia. HIRAID was implemented using a multipronged behaviour change intervention. Data of 920 patients (374 pre-cohort and 546 post-cohort) who had an episode of deterioration within 72 hours of emergency department departure were collected. Statistical tests were conducted as two-sided, with a 95\% confidence interval to determine pre/post cohort association. The StaRI checklist was used in reporting this study.

Results: Patients in the post group were older with more comorbidities, but experienced fewer episodes of clinical deterioration associated with care delivered in the emergency department ( $27 \%$ to $13 \%)$. There was a reduction in treatment delays [pre $n=28(28.3 \%)$ versus post $n=11(15.1 \%), p=0.041,95 \% \mathrm{Cl}(1.1 \%-$ $25.3 \%)$ ], and delay or failure to escalate care when abnormal vital signs were identified [pre $n=20(20.2 \%)$ versus post $\mathrm{n}=5(6.9 \%), \mathrm{p}=0.014,95 \% \mathrm{Cl}(3.5 \%-23.1 \%)]$. The proportion of isolated nursing-related causal factors decreased from $20(21 \%)$ to $6(8 \%)$.

Conclusions: HIRAID is a standardised emergency nursing framework which provides a structured, evidence-based approach to emergency nursing assessment that could be adapted for a variety of clinical settings. Implementing HIRAID using the principles of behavior change is associated with a reduction in clinical deterioration related to emergency care.

\section{Contributions To The Literature}

- The use of HIRAID, a standardised emergency nursing framework is associated with a reduction in clinical deterioration related to emergency care.

- HIRAID use resulted in increased escalation of patient deterioration and time to treatment.

- HIRAID provides a structured approach to application of expert knowledge and skills in the emergency care environment and could be readily adapted for implementation in other jurisdictions and clinical settings using the HIRAID implementation tool-kit.

\section{Introduction}

Recognition and response to deteriorating patients is fundamental to safe, high quality healthcare (1). The emergency department context is a uniquely challenging environment with increased risk of unrecognised or unreported clinical deterioration (2). Initial and ongoing patient assessment, symptom 
control and management are core emergency nursing responsibilities and directly linked to patient safety. Emergency nurses provide care for undiagnosed patients from all age groups, with different levels of illness and injury severity, and fluctuating symptoms and clinical states (2). Emergency nurses are the first clinicians patients see when attending an emergency department, so patient safety is highly dependent on their accurate assessment, interpretation of clinical data, intervention and escalation of care (3). In 2018-19, Australia's 287 emergency departments provided care for more than 8.4 million patients, or 23,000 patients each day (4). Yet, emergency nurses' approach to patient assessment across Australia's emergency departments is inconsistent and results in significant unwarranted variation in nursing care, avoidable patient deterioration, poor pain management, poor nursing documentation, human suffering and patient dissatisfaction $(5,6)$.

In 2019, the New South Wales (NSW) Clinical Excellence Commission reported a $29 \%$ increase in hospital adverse events (AEs) with poor observations and monitoring as a causal factor (7). Undetected clinical deterioration in Australian emergency departments occurs in up to one in seven patients causing highmortality adverse events (8-11). Recognising and responding to deteriorating emergency department patients in a timely manner is primarily an emergency nursing responsibility (12). Failures in recognising and responding to deteriorating ED patients is associated with increased risk of high-mortality adverse events both (cardiac arrest, unplanned intensive care unit (ICU) admission) both during ED care but also in the first 72 hours of hospital admission $(5,6)$. Further, recognition and response to deteriorating ED patients is an Australian emergency care research priority $(13,14)$.

Frequently used patient assessment frameworks such as body systems and vital signs, are not evidencebased and not grounded in patient safety (15). The primary survey (assessment of airway, breathing and circulation) is evidence-based and promotes a focused assessment commensurate with the reliability of specific data to enable recognition of actual or risk of deterioration (15). When patients first attend the emergency department, the triage nurse performs a rapid primary survey and focused physical assessment to determine clinical urgency and the maximum ideal time to emergency care. However, a more comprehensive and standardised patient assessment during emergency care inclusive of the primary survey and vital signs is required (15).

In addition to a comprehensive assessment, emergency nurses must initiate appropriate patient targeted care such as initiating investigations (e.g. pathology tests, diagnostic imaging) and interventions (e.g. pain relief). The quality and timeliness of emergency nurses' care is critical as emergency department (ED) patients often have prolonged waiting times as a result of access block and ED overcrowding. We propose an emergency nursing care delivery solution for any patient presentation called HIRAID (History, Identify Red flags, Assessment, Interventions, Diagnostics, communication and reassessment) (16). This evidence-based patient assessment framework was intended to provide less experienced emergency nurses undertaking postgraduate studies in emergency nursing with a structured approach to patient assessment (17) and has since been revised to expand its utility in ED and reflect the current evidence base (16). HIRAID is the only validated emergency nursing framework for systematic and structured patient assessment (18) (Fig. 1). 
The use of HIRAID improved emergency nurse's detection of clinical indicators of urgency, prioritisation and initiation of treatment, and quality of clinical handover in a simulation study (19). Nursing and medical staff report HIRAID to be a useful tool to improve consistency of patient assessment, quality of documentation and clinical handover (20).

The aim of this study was to determine if the implementation of the HIRAID patient assessment framework improves the safety of emergency care. We hypothesised that after the implementation of HIRAID the proportion of patient deterioration calls related to emergency nursing care within 72 hours of ward admission would decrease.

\section{Methods}

This quasi-experimental pre-post study was conducted between November 2017 and February 2019 in two regional referral hospitals in [Anonymised], Australia. There are two small emergency departments in the health district that transfer patients requiring higher level care to the two larger sites. All emergency departments received the intervention over a 3 month implementation period from October 2017. The smallest site treats 13,000 + patients per year and has two nurses and one general practitioner working per shift. The larger site has a total 110 nurses and 46 medical staff $(65,000+$ presentations per year). This study was conducted per the National Statement on the Conduct of Human Research by the Australian National Health and Medical Research Council and was approved by the site human research ethics committee (Approval Number: 2016/1006).

\section{The intervention implementation}

HIRAID was introduced as a mandatory tool for all nurses working in all four emergency departments using a detailed implementation strategy reported elsewhere (20). The strategy was designed following a survey of the facilitators and barriers to implementation (21). The barriers were mapped to behaviour change techniques using the behaviour change wheel, and assessed using the APEASE criteria (Affordability, Practicability, Effectiveness and cost-effectiveness, Acceptability, Side-effects/safety and Equity) by the ED nurse managers and educators to choose strategies most suitable for each ED (22). Six intervention functions were selected to optimise the implementation of the HIRAID assessment framework: 'training'; 'education'; 'environmental restructuring'; 'enablement'; 'persuasion' and; 'modelling'. Modes of delivery selected for the successful implementation of HIRAID included; a compulsory eLearning module; half day HIRAID workshop; integration of HIRAID into emergency department orientation; initial nursing documentation audits (10 per quarter); and mandatory use of a template for nursing documentation based on the HIRAID assessment framework (20). The uptake of HIRAID was successful. Most nurses in implementation evaluation believed HIRAID reflects their responsibilities as an emergency nurse and $96 \%$ indicated they were using HIRAID. Reasons cited most often were because it was a mandatory requirement, a useful assessment and documentation tool, was easy to use and provided clinical consistency (20). The modes of delivery for HIRAID implementation most useful were HIRAID documentation templates, formal workshop and bedside training. 


\section{Patient identification}

Patients eligible for inclusion were admitted to one of the study sites via the emergency department and received a rapid response call, cardiac arrest call or unplanned ICU admission within 72 hours of admission. To identify these patients, data were obtained from the site rapid response team database. Both sites employ a nurse to identify and audit all patients who receive a rapid response call, cardiac arrest calls or an unplanned ICU admission. Staff can activate a rapid response call when they are concerned patient deterioration needs immediate medical review by the critical care team.

\section{Data collection}

Data were collected from two time periods. The pre period (March 2016-February 2017), which was followed by a 12-month implementation consolidation period, then the post period (March 2018-February 2019). Three clinical nurse consultants independent to the research team collected the data. The nurse consultants had expertise in clinical peer review, rapid response team leadership as well as current critical care, emergency and coronary care experience. The nurses reviewed each patient medical record to extract the required data. Where the patient medical record was not available electronically, the hard copy records were obtained from the medical records department. These data included over 100 event details in eight sections. The sections included patient factors (such as pre-existing conditions calculated using the Charlson Co-morbidity Index (23), vital signs at the time of deterioration call, other hospital services involved in patient's care, factors contributing to care delivery problems, and patient outcomes. A data dictionary was developed in consultation with the nurse consultants and to ensure consistency, where a definition was not already available, a group decision was made and recorded.

\section{Causal factors classification}

To identify influencing or causal factors for clinical deterioration, human factors that may have played a role in the patient deterioration event were collected per the Human Factors Classification Framework for patient safety $(24,25)$ (Table 1$)$. 
Table 1

Classification of factors contributing to the care delivery problem using the Human Factors Classification Framework for patient safety

\begin{tabular}{|lll|}
\hline Domain & Description \\
\hline 1 & Equipment & $\begin{array}{l}\text { Events that involved subcategories of equipment or device failures, } \\
\text { breakages or malfunctions, lack of medical equipment and medical supplies. }\end{array}$ \\
\hline 3 & $\begin{array}{l}\text { Work } \\
\text { Environment }\end{array}$ & $\begin{array}{l}\text { Events resulting from the location of the incident that could not have been } \\
\text { changed by personnel at the time, including lighting, temperature, noise and } \\
\text { physical layout. }\end{array}$ \\
\hline 4 & $\begin{array}{l}\text { Patient } \\
\text { Referred to as staff action events. These resulted from direct involvement by } \\
\text { a staff member, including subcategories of communication failures and } \\
\text { documentation issues, medical task failures, problems monitoring a patient's } \\
\text { status, delays in patient treatment, misdiagnosis of a patient's health } \\
\text { condition, and medication-related issues. }\end{array}$ \\
\hline 5 & $\begin{array}{l}\text { Organisational } \\
\text { Factors }\end{array}$ & $\begin{array}{l}\text { Events resulting from direct involvement by a patient that influenced the } \\
\text { events. }\end{array}$ \\
\hline 6 & $\begin{array}{l}\text { Organisational aspects that directly or indirectly influenced safety and quality } \\
\text { of medical and nursing activities and their management, including work } \\
\text { practices, policies or guidelines, supervision, available resources (including } \\
\text { staffing and equipment), work pressure and other organisational factors. }\end{array}$ \\
\hline 7 & $\begin{array}{l}\text { Individual } \\
\text { Factors }\end{array}$ & $\begin{array}{l}\text { Oharacteristics of staff members, including knowledge and skills, experience, } \\
\text { stress, fatigue and other individual factors. }\end{array}$ \\
\hline & \begin{tabular}{l} 
Additional events not elsewhere classified. \\
\hline
\end{tabular} &
\end{tabular}

The Human Factors Classification Framework was selected on the basis of high inter-rater reliability (24) and its basis on James Reason's model of organisational incidents (26). Error was classified using Rasmussen's framework (27) of skill, rule or knowledge-based errors classifications whereby a skill-based errors were defined as an unintentional failure in the execution of a well-rehearsed action or routine task that required little conscious attention; rule-based errors were defined as unintentional failures during activities conducted in familiar situations controlled by stored rules; and knowledge-based errors were defined as unintentional failures during a novel situation that required conscious analytic processing and stored knowledge (27). Violations were defined as an intentional failure to follow accepted work practices, guidelines or procedures during the execution of a task, a violation does not indicate the intent to cause harm (28).

For a deterioration event to be labelled as related to care received in the emergency department, the causal factors and/or errors identified must have a clear relationship with or contribution to the deterioration event. Examples included "Staff Action"; delay to intravenous antibiotics in a septic patient, missed diagnostic information like an arterial blood gas in a deteriorating respiratory patient, progressive deterioration in emergency with no identification or escalation. In contrast, if on day two of admission the patient suddenly had an unexpected cardiac arrest, or a rapid response call for a post-op complication, if 
these events had no indication to treatment and care provided within emergency department it would not be considered as a failure in emergency department.

\section{Data management and analysis}

The majority ( $86 \%$ ) of cases were reviewed by the one auditor. To ensure consistency in allocation of causal factors, one of the auditors performed a screen of every case. Where there was any uncertainty, a group discussion was held until consensus was reached. Data were cleaned (validations and definitions) and integrated for an initial descriptive analysis. Data were analysed using Stata Version 14.2 (StataCorp, College Station, USA) to test if the groups were equal in characteristics. Continuous variables were compared using t-tests or Mann Whitney $\mathrm{U}$ tests and categorical variables were compared using Chisquare tests. Two-sample test of proportions used to assess the change of proportions pre and post and were also used to determine whether there a statistical difference in the numbers of cases included in the final pre and post cohorts. All statistical tests were conducted as two-tailed, and a confidence level of $95 \%$ was used to determine if there was a significant association between the pre/post cohorts and study variables of interest. The StaRI checklist was used in reporting this study.

\section{Results}

There were 374 patients in the pre-intervention cohort and 546 in the post-intervention cohort eligible for inclusion as they deteriorated on the ward within 72 hours of admission via one of the study site EDs (Fig. 2). In the pre period there were 100,501 presentations to and 32,048 admissions via the ED. In the post period, ED presentations increased by $11.49 \%$ to 112,048 and admissions via the ED increased by $6.33 \%$ to 34,078 . Hospital bed occupancy was $84 \%$ pre and $83 \%$ post.

Patients in the post group were older ( $75 \mathrm{yrs}$ vs $81 \mathrm{yrs}, \mathrm{p}=.014)$ with more comorbidities (4.32 vs $5.79, \mathrm{p}$ $<0.001)$ and higher incidence of polypharmacy $(49 \%$ vs $77 \%, p<0.001)$. The post group also had a higher proportion of patients from culturally and linguistically diverse backgrounds $(2 \%$ vs $8 \%, p=0.019)$ and patients who had re-presented for the same condition ( $2 \%$ vs $12 \%, p<0.001)$. There was a low incidence and no difference between groups identified for the following characteristics: aggression $(p=1.000)$, substance misuse $(p=1.000)$, mental illness $(p=0.184)$, and delirium $(p=0.136)$. There was no significant difference in gender $(p=0.346)$, ED length of stay (LOS) $(p=0.477)$ or at which emergency department the patient was treated $(p=0.908)$ (Table 2). 
Table 2

Comparison of patient characteristics (pre/post) with clinical deterioration with 72 hours of emergency department departure relating to emergency care

\begin{tabular}{|c|c|c|c|}
\hline Variable & Pre $n=(101)$ * & Post $(n=73)$ & Statistic ${ }^{\star *}$ \\
\hline Age - Median (IQR) & $75(58-85)$ & $81(69-87)$ & 0.014 \\
\hline Gender - n (\%) & $43(43.4)$ & $37(50.7)$ & 0.346 \\
\hline Female & $56(56.6)$ & $36(49.3)$ & \\
\hline \multicolumn{4}{|l|}{ Male } \\
\hline ED LOS (h) - Median (IQR) & $9.75(5.4-17.0)$ & $8.70(5.4-15.2)$ & 0.477 \\
\hline Site - n (\%) & $74(75.8)$ & $54(74.0)$ & 0.908 \\
\hline Site 1 & $25(25.3)$ & $19(26.0)$ & \\
\hline \multicolumn{4}{|l|}{ Site 2} \\
\hline $\mathrm{CCl}$ - mean (SD) & $4.32(2.6)$ & $5.79(3.0)$ & $<0.001$ \\
\hline Polypharmacy - n (\%) & $49(50.0)$ & $56(76.7)$ & $<0.001$ \\
\hline Time of presentation - $\mathrm{n}(\%)$ & $46(46.5)$ & $28(38.4)$ & 0.563 \\
\hline Morning (07:00-15:00) & $31(31.3)$ & $27(37.0)$ & \\
\hline Afternoon (15:01-22:00) & $22(22.2)$ & $18(24.7)$ & \\
\hline \multicolumn{4}{|l|}{ Night (22:01-06:59) } \\
\hline Time of admission - $\mathrm{n}(\%)$ & $31(31.3)$ & $21(28.8)$ & 0.007 \\
\hline Morning (07:00-15:00) & $38(38.4)$ & $43(58.9)$ & \\
\hline Afternoon (15:01-22:00) & $30(30.3)$ & $9(12.3)$ & \\
\hline \multicolumn{4}{|l|}{ Night (22:01-06:59) } \\
\hline \multicolumn{4}{|c|}{ * Total number were 101 with some variables containing missing values. } \\
\hline \multicolumn{4}{|c|}{ 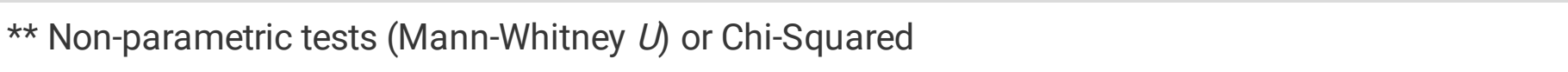 } \\
\hline $\begin{array}{l}\text { IQR: Interquartile Range, CCl: } \\
\text { Stay, h: Hours, SD: Standard }\end{array}$ & morbidity Inde & S: Emergency & nt Length \\
\hline
\end{tabular}

Although there were more episodes of clinical deterioration within 72 hours of emergency admission for the post group, there were fewer in-hospital cardiac arrests (pre $n=9,9 \%$ vs post $n=0$ ) and unplanned ICU admissions (pre $n=19,18.2 \%$ vs post $n=8,10.8 \%$ ). There was an increase in the proportion of rapid response calls (pre $n=71,71.7 \%$ vs post $n=65,89.0 \% p=0.007$ ). There was no difference in rapid response call triggers however the median (IQR) time from emergency department discharge to the 
deterioration event decreased from 19.5 hours (7.20-36.17) to 11 hours $(4.62-20.03), p=0.005)$. More patients died following their deterioration event in the post period ( $7 \%$ vs $22 \%, p=0.005)$ (Table 3 ).

Table 3

Number and patient physiologic parameter characteristics of deterioration episode

\begin{tabular}{|llll|}
\hline Variable & Pre $\mathbf{n}=(\mathbf{1 0 1}) *$ & Post $(\mathbf{n}=\mathbf{7 3})$ & Statistic** \\
\hline Call type - n (\%) & $9(9.09)$ & $0(0.00)$ & 0.007 \\
Cardiac arrest & $71(71.72)$ & $65(89.04)$ & \\
PACE 2 & $19(18.19)$ & $8(10.96)$ & \\
Unplanned ICU admit & & & \\
\hline Vital signs & $21.50(17.00-$ & $21.5(17.0-30.0)$ & 0.863 \\
RR - Median (IQR) & $32.50)$ & $94.0(90.0-96.0)$ & 0.505 \\
SpO2 - Median (IQR) & $95.0(86.0-97.0)$ & $93.5(78.5-112.5)$ & 0.234 \\
HR - Median (IQR) & $104.0(70.0-128.0)$ & $117.0(87.0-$ & 0.239 \\
SBP - Median (IQR) & $130.0(90.0-161.0)$ & $144.0)$ & 0.732 \\
DBP - Median (IQR) & $71.5(48.0-86.0)$ & $68.0(52.0-80.0)$ & 0.254 \\
AVPU - n (\%) & $41(59.4)$ & $44(67.7)$ & 0.349 \\
A & $3(4.2)$ & $6(9.2)$ & 0.266 \\
P & $18(26.1)$ & $9(13.9)$ & 0.750 \\
U & $7(10.1)$ & $6(9.2)$ & \\
V & $14.0(8.0-15.0)$ & $14.5(11.0-15.0)$ & \\
GCS - Median (IQR) & $3.5(0.0-7.5)$ & $0.0(0.0-5.0)$ & \\
Pain Score (/10) - Median (IQR) & $7.4(6.2-9.8)$ & $7.2(5.7-9.9)$ & \\
BGL - Median (IQR) & & & \\
\hline Time from ED discharge to call - Median & $19.5(7.2-36.2)$ & $10.6(4.6-20.0)$ & 0.005 \\
(IQR) & & & \\
\hline$*$ TR & & \\
\hline
\end{tabular}

* Total number were 101 with some variables containing missing values.

** Non-parametric tests (Mann-Whitney $U$ ) or Chi-Squared

RR: Respiratory Rate, Sp02: Oxygen saturation, HR: Heart Rate, SBP: Systolic Blood Pressure, DBP: Diastolic Blood Pressure, AVPU: Alert, Visual, Pain, Unresponsive, GCS: Glasgow Coma Score, BGL: Blood Glucose Level, ED: Emergency Department, IQR: Interquartile Range, SD: Standard Deviation.

\section{Causal Factors for Clinical Deterioration}


The proportion of deterioration episodes related to emergency department care decreased by half [pre $\mathrm{n}=$ $101(27.0 \%)$ to post $\mathrm{n}=73(13.4 \%), \mathrm{p}<0.001,95 \% \mathrm{Cl}(8.5 \%-18.8 \%)]$. There was a reduction in treatment delays [pre $\mathrm{n}=28(28.3 \%)$ vs post $\mathrm{n}=11(15.1 \%), \mathrm{p}=0.041,95 \% \mathrm{Cl}(1.1 \%-25.3 \%)$ ], delay in diagnoses [pre $n=15(15.2 \%)$ vs post $n=3(4.1 \%), p=0.019,95 \% \mathrm{Cl}(2.6 \%-19.4 \%)$ ], delay or failure to escalate care when abnormal vital signs were identified [pre $n=20(20.2 \%)$ vs post $n=5(6.9 \%), p=0.014,95 \% \mathrm{Cl}(3.5 \%$ $-23.1 \%)$ ]. There was a reduction in the number of organisational factors from 99 to 73 [pre $n=64$ $(64.6 \%)$ post $\mathrm{n}=19(26.0 \%), \mathrm{p}<0.001,95 \% \mathrm{Cl}(24.8-52.4)]$. When determining if the causal factor was primarily nursing, medical or nursing and medical related, the number and proportion of isolated nursing related causal factors decreased from $20(21 \%)$ to $6(8 \%)$. The number and proportion of isolated nursing related causal factors decreased from $20(21 \%)$ to $6(8 \%)$ (Table 4$)$. 
Table 4

Causal factors of deterioration episode

\begin{tabular}{|c|c|c|c|c|}
\hline ED problems & Pre & Post & $\begin{array}{l}\mathrm{p}- \\
\text { value }\end{array}$ & $\begin{array}{l}\text { Confidence interval of the } \\
\text { difference in proportions (\%) }\end{array}$ \\
\hline $\begin{array}{l}\text { WH ED problems (number of } \\
\text { deterioration }<72 \mathrm{hrs} \text { ) }\end{array}$ & $\begin{array}{l}76 \\
(758)\end{array}$ & $\begin{array}{l}54 \\
(1141)\end{array}$ & \multirow[t]{2}{*}{$\dot{0} 001$} & \multirow[t]{2}{*}{$3.0-7.6$} \\
\hline Proportion & $10.0 \%$ & $4.7 \%$ & & \\
\hline $\begin{array}{l}\text { SDMH ED problems (number of } \\
\text { deterioration }<72 \mathrm{hrs} \text { ) }\end{array}$ & $\begin{array}{l}25 \\
(316)\end{array}$ & $\begin{array}{l}19 \\
(459)\end{array}$ & \multirow[t]{2}{*}{0.026} & \multirow[t]{2}{*}{$0.5-7.1$} \\
\hline Proportion & $7.9 \%$ & $4.1 \%$ & & \\
\hline $\begin{array}{l}\text { Overall ED problems (number of } \\
\text { deterioration }<72 \mathrm{hrs} \text { ) }\end{array}$ & $\begin{array}{l}101 \\
(374)\end{array}$ & $\begin{array}{l}73 \\
(546)\end{array}$ & \multirow[t]{2}{*}{$<.001$} & \multirow[t]{2}{*}{$8.5-18.8$} \\
\hline Proportion & $27.0 \%$ & $13.4 \%$ & & \\
\hline \multicolumn{5}{|l|}{ Equipment $(n 1=100, n 2=73)-n(\%)$} \\
\hline Equipment & \multirow{2}{*}{$\begin{array}{l}9 \\
(8.1)\end{array}$} & \multirow{2}{*}{8} & 0.521 & $-11.8-6.1$ \\
\hline Medical Equipment & & & \multirow{3}{*}{$\begin{array}{l}0.082 \\
0.389\end{array}$} & \multirow{3}{*}{$\begin{array}{l}0.16-7.9 \\
-1.0-3.0\end{array}$} \\
\hline Medical Supply & $\begin{array}{l}4 \\
(4.0)\end{array}$ & $\begin{array}{l}0(0.0) \\
0(0.0)\end{array}$ & & \\
\hline & $\begin{array}{l}1 \\
(1.0)\end{array}$ & & & \\
\hline \multicolumn{5}{|c|}{ Staff action overall $(n 1=99 n 2=73)-n(\%)$} \\
\hline Staff action overall & $\begin{array}{l}95 \\
(96.0)\end{array}$ & $\begin{array}{l}68 \\
(95.8)\end{array}$ & 0.952 & $-5.9-6.3$ \\
\hline Recognition of red flags & \multirow{2}{*}{$\begin{array}{l}20 \\
(20.2)\end{array}$} & \multirow{2}{*}{$\begin{array}{l}16 \\
(21.9)\end{array}$} & 0.785 & $-14.1-10.6$ \\
\hline Delay in treatment & & & 0.041 & $1.1-25.3$ \\
\hline $\begin{array}{l}\text { delay recognition of resuscitation } \\
\text { plan }\end{array}$ & $\begin{array}{l}28 \\
(28.3)\end{array}$ & $\begin{array}{l}11 \\
(15.1)\end{array}$ & 0.041 & $-27.0--0.4$ \\
\hline Delay recognition to sepsis & \multirow{2}{*}{$\begin{array}{l}19 \\
(19.2)\end{array}$} & \multirow{2}{*}{$\begin{array}{l}24 \\
(32.9)\end{array}$} & 0.759 & $-11.8-8.6$ \\
\hline Communication - ctaff to nation & & & 0.372 & $-12.7-4.9$ \\
\hline Medical Management error & $\begin{array}{l}12 \\
(12.1)\end{array}$ & $\begin{array}{l}10 \\
(13.7)\end{array}$ & 0.073 & $-27.6-1.3$ \\
\hline Senior medical input & \multirow{2}{*}{$\begin{array}{l}7 \\
(7.1)\end{array}$} & \multirow{2}{*}{$\begin{array}{l}8 \\
(11.0)\end{array}$} & 0.618 & $-9.0-5.4$ \\
\hline Concultation reauects & & & 0.841 & $-7.6-9.3$ \\
\hline Conflicting patient care & $\begin{array}{l}29 \\
(29.3)\end{array}$ & $\begin{array}{l}31 \\
(42.5)\end{array}$ & 0.210 & $-17.4-4.0$ \\
\hline Delay in diagnosis & \multirow{2}{*}{$\begin{array}{l}5 \\
(5.1)\end{array}$} & \multirow{3}{*}{$\begin{array}{l}5(6.9) \\
6(8.2)\end{array}$} & 0.019 & $2.6-19.4$ \\
\hline Nursing Management error & & & 0.226 & $-21.8-5.2$ \\
\hline Misdiagnosis & $\begin{array}{l}9 \\
(8.2)\end{array}$ & & 0.051 & $0.7-12.7$ \\
\hline
\end{tabular}




\begin{tabular}{|c|c|c|c|c|}
\hline $\begin{array}{l}\text { EBmmunication staff to staff } \\
\text { Documentation - medical }\end{array}$ & Pre & Pogst) & $\begin{array}{l}0.653 \\
\text { palye } \\
\text { y.jy4 }\end{array}$ & $\begin{array}{l}\text { - } 8 \text { onfidence interval of the } \\
\text { difference in proportions (\%) }\end{array}$ \\
\hline Documentation - nursing & $\begin{array}{l}11 \\
(11.1)\end{array}$ & $3(4.1)$ & 0.267 & $-4.7-17.7$ \\
\hline Monitoring of observations & & $(31.5)$ & 0.207 & $-2.0-10.6$ \\
\hline Delay or failure to escalate & $(15.2)$ & $1(1.4)$ & 0.014 & $3.5-23.1$ \\
\hline Monitoring blood results & $\begin{array}{l}23 \\
(23.2)\end{array}$ & 29 & 0.341 & $-14.3-5.1$ \\
\hline Alternate chart commenced & 8 & & 0.303 & $-2.0-7.4$ \\
\hline Which chart? & $(0.1)$ & $(32.9)$ & 0.207 & $-2.0-10.6$ \\
\hline Delays to transfer & $(36.4)$ & 10 & 0.508 & $-4.9-10.1$ \\
\hline Safety/Security check & 26 & & 0.222 & $-0.8-4.8$ \\
\hline Other human factors & $(20.3)$ & $2(2.1)$ & 0.243 & $-4.0-1.3$ \\
\hline \multirow[t]{12}{*}{ Consumer involvement } & $(20.2)$ & (0.0) & \multirow[t]{11}{*}{0.247} & \multirow[t]{11}{*}{$-11.6-3.2$} \\
\hline & \multirow{2}{*}{$\begin{array}{l}7 \\
(7.1)\end{array}$} & $(13.7)$ & & \\
\hline & & $1(1.4)$ & & \\
\hline & $\begin{array}{l}20 \\
(20.2)\end{array}$ & $2(2.7)$ & & \\
\hline & \multirow{2}{*}{$\begin{array}{l}9 \\
(9.1)\end{array}$} & $4(5.5)$ & & \\
\hline & & $0(0.0)$ & & \\
\hline & $\begin{array}{l}4 \\
(4.0)\end{array}$ & $1(1.4)$ & & \\
\hline & $\begin{array}{l}7 \\
(7.1)\end{array}$ & $6(8.2)$ & & \\
\hline & $\begin{array}{l}8 \\
(8.1)\end{array}$ & & & \\
\hline & $\begin{array}{l}2 \\
(2.0)\end{array}$ & & & \\
\hline & $\begin{array}{l}0 \\
(0.0)\end{array}$ & & & \\
\hline & $\begin{array}{l}4 \\
(4.0)\end{array}$ & & & \\
\hline Organisational factors $(\mathrm{n} 1=9$ & 3) $-n(9$ & & & \\
\hline
\end{tabular}




\begin{tabular}{|c|c|c|c|c|}
\hline ED problems & Pre & Post & $\begin{array}{l}\mathrm{p}- \\
\text { value }\end{array}$ & $\begin{array}{l}\text { Confidence interval of the } \\
\text { difference in proportions (\%) }\end{array}$ \\
\hline \multirow{7}{*}{$\begin{array}{l}\text { Supervision } \\
\text { Work Practice related } \\
\text { Policy/guideline not followed } \\
\text { Policy/guideline unclear } \\
\text { Staffing issues } \\
\text { Delay in transfer }\end{array}$} & \multirow{2}{*}{$\begin{array}{l}0 \\
(0.0)\end{array}$} & $1(1.4)$ & 0.243 & $-4.0-1.3$ \\
\hline & & $1(55)$ & 0658 & $-70-51$ \\
\hline & $\begin{array}{l}4 \\
(4.0)\end{array}$ & & ח ०८8 & $-168-15$ \\
\hline & \multirow{2}{*}{$\begin{array}{l}6 \\
(6.1)\end{array}$} & $(13.7)$ & 0.080 & $-256-16$ \\
\hline & & 25 & תח? & 007 \\
\hline & \multirow{2}{*}{$\begin{array}{l}22 \\
(22.2)\end{array}$} & & 0.000 & $-2.0-1.4$ \\
\hline & & $1(1.4)$ & 0.044 & $1.0-17.1$ \\
\hline & $\begin{array}{l}4 \\
(4.0)\end{array}$ & $3(4.1)$ & & \\
\hline & $\begin{array}{l}13 \\
(13.1)\end{array}$ & & & \\
\hline \multicolumn{5}{|c|}{ Individual factors $(\mathrm{n} 1=99, \mathrm{n} 2=73)-\mathrm{n}(\%)$} \\
\hline Staff training & \multirow{2}{*}{$\begin{array}{l}0 \\
(0.0)\end{array}$} & $5(6.8)$ & 0.008 & $-12.6--1.1$ \\
\hline Staff experience & & $3(4.1)$ & 0.570 & $-4.6-8.5$ \\
\hline \multirow[t]{2}{*}{ Staff stress } & $\begin{array}{l}6 \\
(6.1)\end{array}$ & $0(0.0)$ & 0.222 & $-0.8-4.8$ \\
\hline & $\begin{array}{l}2 \\
(2.0)\end{array}$ & & & \\
\hline \multicolumn{5}{|l|}{ Error $(\mathrm{n} 1=99, \mathrm{n} 2=73)-\mathrm{n}(\%)$} \\
\hline \multirow{3}{*}{$\begin{array}{l}\text { Rule based error } \\
\text { Knowledge based error } \\
\text { Skill based error }\end{array}$} & \multirow{2}{*}{$\begin{array}{l}55 \\
(55.6)\end{array}$} & \multirow{2}{*}{$\begin{array}{l}51 \\
(69.9)\end{array}$} & 0.057 & $-28.7-0.0$ \\
\hline & & & 0.487 & $-8.8-18.7$ \\
\hline & $\begin{array}{l}32 \\
(32.3)\end{array}$ & $(27.4)$ & 0.124 & $-0.7-10.1$ \\
\hline \multirow{3}{*}{$\begin{array}{l}\text { Violation error } \\
\text { Nil error }\end{array}$} & $\begin{array}{l}6 \\
(6.1)\end{array}$ & $1(1.4)$ & \multirow{2}{*}{$\begin{array}{l}< \\
0.001 \\
0.508\end{array}$} & $24.8-52.4$ \\
\hline & $\begin{array}{l}64 \\
(64.6)\end{array}$ & $\begin{array}{l}19 \\
(26.0)\end{array}$ & & $-4.9-10.1$ \\
\hline & $\begin{array}{l}(04.0) \\
8 \\
(8.1)\end{array}$ & $4(5.5)$ & & \\
\hline
\end{tabular}

\section{Discussion}

In this study implementation of HIRAID in the emergency care setting improved patient safety as evidenced by a reduction in patient deterioration related to emergency care within 72 hours of admission via the emergency department. This is likely due to the demonstrated increased escalation of patient deterioration and time to treatment, which are key components of the HIRAID framework. 
HIRAID has a focus on obtaining relevant and important information to guide assessment, clinical intervention, decision making and clinical handover (16). Clinical handover is a safety-critical communication event and accurate communication during clinical handover is a global patient safety priority (29). Ineffective communication and communication failures during clinical handover increases the risk of adverse events and patient-related errors (30). A structured communication process, increased self-efficacy (31) and skills in graded assertiveness (19) may have contributed to the reduction in failure of escalation as a casual factor to patient deterioration in this study. Further, the reduction in deterioration attributed to overall emergency department care suggests that the improved assessment and management of nurses may have a flow on effect to other emergency staff. The ED is a dynamic, stressful context of practice, where interprofessional interdependence and collaboration are vital. In previous evaluations of HIRAID, medical officers have reported improved quality of clinical handover when compared to previously used tools. Collaborative interprofessional relationships and effective communication between ED medical and nursing staff are vital to patient safety and high quality emergency care (32).

The robustness of the implementation strategy design and development contributed to the high uptake and application of HIRAID. The implementation strategy addressed the complexity of the emergency care environment and was strongly supported by organisational key stakeholders (33). There are multiple models available on which to develop and plan an implementation strategy (34). However, the sustained success of any change is dependent on human behaviour, so this is a key consideration in any implementation strategy. Although all emergency nurses at the study sites were required to use HIRAID, they were also empowered with the capability, opportunity and motivation to do so. Most respondents in a multicentre survey believed their fundamental responsibility as an emergency nurse is to ensure patient safety and that HIRAID provided a mechanism to enable this (20).

The HIRAID emergency nursing assessment framework and implementation strategy can be adapted for other practice settings, is complimentary to existing emergency nursing courses and does not rely on significant upskilling programs. (1). However, HIRAID has only been formally tested in Australia, so implementation in other countries requires consideration of scope of practice and resources, formal consultation with peak bodies and context-specific evaluation. A cost-benefit analysis would also be of value.

This study was a pre post study with a 12-month implementation period between data collection periods so there is a possibility that processes of care changed over time. This risk was managed by monitoring and confirmation with key stakeholders (nurse managers and educators) that there was no other formal intervention or assessment methods implemented during the study period. The study data were obtained from organisational databases and medical records, thus there was potential for data error and missing data. Further, organisational and record data do not enable a detailed understanding of clinician characteristics that may influence application of the HIRAID framework and therefore processes of assessment and management. This risk was actively managed by a detailed analysis of barriers and facilitators to implementation of HIRAID and tailored implementation at each site. It is possible there was 
a Hawthorne effect in the post intervention arm, although as this change to clinical practice was mandated, this is not likely. Finally, the study was conducted in regional Australia, so the generalisability of the study findings to other jurisdictions may be limited. A randomised control study that incorporates consumers and an economic evaluation would provide the necessary evidence for embedding HIRAID into policy and practice for system-wide change.

\section{Conclusions}

Initial and ongoing patient assessment, symptom control and management are core emergency nursing responsibilities and directly linked to patient safety. Failures in recognising and responding to deteriorating ED patients is associated with high-mortality adverse events such as cardiac arrest and unplanned ICU admission.

HIRAID is a validated framework designed to provide emergency nurses with a structured and systematic approach to patient assessment and management. The use of HIRAID is associated with a reduction in clinical deterioration related to emergency care by enhancing nursing practice through increased escalation of patient deterioration. The reasons for this reduction can be explained by a reduction in the proportion of causal factors relating to nurse action, violation related errors (intentional failure to follow accepted work practices, guidelines, for example, where it has become routine to practice in a certain way), treatment delays and failures in escalation of clinical deterioration. The evidence-based nature of the HIRAID framework and implementation strategy means HIRAID is readily adaptable for implementation in other jurisdictions or contexts of practice.

\section{Abbreviations}

- AEs: Adverse events

- APEASE: Affordability, Practicability, Effectiveness and cost-effectiveness, Acceptability, Sideeffects/safety and Equity

- AVPU: Alert, Visual, Pain, Unresponsive

- BGL: Blood Glucose Level

- CCl: Charlson Comorbidity Index

- DBP: Diastolic Blood Pressure

- ED: Emergency Department

- GCS: Glasgow Coma Score

- HIRAID: History, Identify Red flags, Assessment, Interventions, Diagnostics, communication and reassessment

- HR: Heart Rate

- ICU: Intensive Care Unit

- IQR: Interquartile Range 
- LOS: Length of Stay

- NSW: New South Wales

- RR: Respiratory Rate,

- SBP: Systolic Blood Pressure

- SD: Standard Deviation

- Sp02: Oxygen saturation

\section{Declarations}

\section{Ethics approval and consent to participate:}

This study was conducted per the National Statement on the Conduct of Human Research by the Australian National Health and Medical Research Council and was approved by the site human research ethics committee (Approval Number: 2016/1006).

Consent for publication:

Not applicable.

Availability of data and materials:

The datasets used and/or analysed during the current study are available from the corresponding author on reasonable request and within ethical approval scope.

Competing interests:

The authors have no conflicts of interest to declare.

Funding:

The New South Wales Health Agency for Clinical Innovation and the Australian College of Nursing provided financial support for the conduct of the research. The funders were not involved in the study design, in the collection, analysis and interpretation of data or writing of the report.

Authors' contributions:

As outlined below all authors meet the formal requirements of authorship established by the International Committee of Medical Journal Editors (ICMJE) in that all authors:

Made substantial contributions to the (a) conception or design of the manuscript; or (b) to the acquisition, analysis, or interpretation of data for the work; or (c) Drafted, or (d) revised the manuscript critically for important intellectual content.

Gave final approval of the version to be published; and 
Agreed to be accountable for all aspects of the work in ensuring that questions related to the accuracy or integrity of any part of the work are appropriately investigated and resolved.

\begin{tabular}{|c|c|c|c|c|c|c|}
\hline \multirow[t]{2}{*}{ AUTHOR } & \multicolumn{6}{|c|}{ ICMJE Author Definition } \\
\hline & $1(\mathrm{a})$ & $1(b)$ & 1 (c) & $1(d)$ & 2 & 3 \\
\hline Kate Curtis & $\diamond$ & $\diamond$ & $\diamond$ & $\diamond$ & $\diamond$ & $\diamond$ \\
\hline Belinda Munroe & $\diamond$ & $\diamond$ & & $\diamond$ & $\diamond$ & $\diamond$ \\
\hline Margaret Fry & & $\diamond$ & & $\diamond$ & $\diamond$ & $\diamond$ \\
\hline Julie Considine & & $\diamond$ & & $\diamond$ & $\diamond$ & $\diamond$ \\
\hline Erin Tuala & & $\diamond$ & & $\diamond$ & $\diamond$ & $\diamond$ \\
\hline Michael Watts & & $\diamond$ & & $\diamond$ & $\diamond$ & $\diamond$ \\
\hline Hatem Alkhouri & & $\diamond$ & & $\diamond$ & $\diamond$ & $\diamond$ \\
\hline Tiana Elphick & & $\diamond$ & & $\diamond$ & $\diamond$ & $\diamond$ \\
\hline Kate Ruperto & & $\diamond$ & & $\diamond$ & $\diamond$ & $\diamond$ \\
\hline Jacquie Barrass & & $\diamond$ & & $\diamond$ & $\diamond$ & $\diamond$ \\
\hline Sharyn Balzer & & $\diamond$ & & $\diamond$ & $\diamond$ & $\diamond$ \\
\hline Bronwynne Chisholm & & $\diamond$ & & $\diamond$ & $\diamond$ & $\diamond$ \\
\hline Connie Van & & $\diamond$ & & $\diamond$ & $\diamond$ & $\diamond$ \\
\hline Ramon Z. Shaban & & $\diamond$ & & $\diamond$ & $\downarrow$ & $\diamond$ \\
\hline
\end{tabular}

Acknowledgements:

Marg Swinton (deceased) - former clinical nurse educator at Shellharbour Hospital dedicated her life to supporting and educating emergency nurses in delivery of quality patient care. Ms Swinton led the implementation of the intervention at Shellharbour emergency department.

\section{References}

1. Curtis K, Brysiewicz P, Shaban RZ, Fry M, Considine J, Gamboa FEA, et al. Nurses responding to the World Health Organization (WHO) priority for emergency care systems for universal health coverage. International Emergency Nursing. 2020;50:100876.

2. Fry M. Chapter 1: Emergency Nursing in Australia and New Zealand. In: Curtis K, Ramsden C, Shaban R, Fry M, Considine J, editors. Emergency and Trauma Care: For Nurses and Paramedics. 3rd ed. Chatswood: Elsevier Australia; 2019. 
3. Fasoli DR. The culture of nursing engagement: a historical perspective. Nursing Administration Quarterly. 2010;34(1):18-29.

4. Australian Institute of Health and Welfare. Emergency department care 2018-19. Canberra; 2020.

5. Considine J, Jones D, Pilcher D, Currey J. Patient physiological status during emergency care and rapid response team or cardiac arrest team activation during early hospital admission. European Journal of Emergency Medicine. 2017;24(5):359-65.

6. Forster AJ, Rose NGW, Van Walraven C, Stiell I. Adverse events following an emergency department visit. Quality Safety in Health Care. 2007;16(1):17-22.

7. Clinical Excellence Commission. System Factors identified in the Clinical Management RCA Review Committee. NSW Government; 2019.

8. Scott BM, Considine J, Botti M. Unreported clinical deterioration inemergency department patients: A point prevalence study. Australasian Emergency Nursing Journal. 2015;18(1):33-41.

9. Lambe K, Currey J, Considine J. Frequency of vital sign assessment and clinical deterioration in an Australian emergency department. Australas Emerg Nurs J. 2016;19(4):217-22.

10. Hosking J, Considine J, Sands N. Recognising clinical deterioration in emergency department patients. Australasian Emergency Nurses Journal. 2014;17(12):59-67.

11. Hogan H, Healey F, Neale G, Thomson R, Vincent C, Black N. Preventable deaths due to problems in care in English acute hospitals: a retrospective case record review study. BMJ Quality Safety. 2012;21:737-45.

12. Considine J, Rhodes K, Jones D, Currey J. Systems for recognition and response to clinical deterioration in Victorian emergency departments. Australasian Emergency Care. 2018;21(1):3-7.

13. Keijzers G, Thom O, Taylor D, Knott J. Clinical research priorities in emergency medicine. Emergency Medicine Australasia. 2014;26(1):19-27.

14. Considine J, Curtis K, Shaban RZ, Fry M. Consensus-based clinical research priorities for emergency nursing in Australia. Australasian Emergency Care. 2018;21(2):43-50.

15. Considine J, Currey J. Ensuring a proactive, evidence-based, patient safety approach to patient assessment. J Clin Nurs. 2014;24(1-2):300-7.

16. Munroe B, Curtis K, Margerat M, Strachan L, Buckley T. HIRAID: An evidence-informed emergency nursing assessment framework. Australasian Emergency Nurses Journal. 2015;18(2):83-97.

17. Curtis K, Murphy M, Hoy S, Lewis $M$. The emergency nursing assessment process - a structured framework for a systematic approach. Australasian Emergency Nurses Journal. 2009;12(4):130-6.

18. Munroe B, Curtis K, Considine J, Buckley T. The Impact Structured Patient Assessment Frameworks Have on Patient Care: An Integrative Review. J Clin Nurs. 2013;22(21-22):2991-3005.

19. Munroe B, Curtis K, Murphy M, Strachan L, Considine J, Hardy J, et al. A structured framework improves clinical patient assessment and non-technical skills of early career emergency nurses: a pre-post study using full immersion simulation. J Clin Nurs. 2016;25(15-16):2262-74. 
20. Curtis K, Munroe B, Van C, Elphick T-L. The implementation and usability of HIRAID, a structured approach to emergency nursing assessment. Australasian Emergency Care. 2020;23(1):62-70.

21. Munroe B, Curtis K, Buckley T, Lewis M, Atkins L. Optimising implementation of a patient-assessment framework for emergency nurses: A mixed-method study. J Clin Nurs. 2018;27(1-2):269-86.

22. Michie S, Atkins L, West R. The Behaviour Change Wheel: a guide to designing interventions. Great Britain: Silverback Publishing; 2014.

23. Quan H, Li B, Couris CM, Fushimi K, Graham P, Hider P, et al. Updating and validating the Charlson comorbidity index and score for risk adjustment in hospital discharge abstracts using data from 6 countries. Am J Epidemiol. 2011;173(6):676-82.

24. Mitchell RJ, Williamson A, Molesworth B. Application of a human factors classification framework for patient safety to identify precursor and contributing factors to adverse clinical incidents in hospital. Appl Ergon. 2016;52:185-95.

25. Curtis K, Mitchell R, McCarthy A, Wilson K, Van C, Kennedy B, et al. Development of the major trauma case review tool. Scandinavian Journal of Trauma, Resuscitation and Emergency Medicine. 2017;25(20).

26. Reason J. Human error: models and management. Bmj. 2000;320(7237):768-70.

27. Rasmussen J. Human errors. A taxonomy for describing human malfunction in industrial installations. Journal of Occupational Accidents. 1982;4(2):311-33.

28. Reason J. Human error: models and management. BMJ: British Medical Journal. 2000;320(7237):768-70.

29. WHO Collaborating Centre for Patient. Safety Solutions Communication During Patient Hand-Overs. Patient safety solutions 2007.

30. Australian Commission on Safety and Quality in Health Care. The NSQHS Standards, Communicating for Safety Standard, Communication at clinical handover Action 6.4b. Canberra2019.

31. Munroe B, Buckley T, Curtis K, Murphy M, Strachan L, Hardy J, et al. The impact of HIRAID on emergency nurses' self-efficacy, anxiety and perceived control: A simulated study. International Emergency Nursing. 2016;25:53-8.

32. Curtis K, Tzannes A, Rudge T. How to talk to Doctors - A guide for effective communication. Int Nurs Rev. 2011;58(1):13-20.

33. Bate P, Mendel P, Robert G. Organizing for quality: the improvement journeys of leading hospitals in Europe and the United States: Radcliffe Publishing; 2008.

34. Schaffer MA, Sandau KE, Diedrick L. Evidence-based practice models for organizational change: overview and practical applications. J Adv Nurs. 2013;69(5):1197-209.

\section{Figures}




\begin{tabular}{|c|c|}
\hline \multirow{5}{*}{ (c) Curtis, Munroe, Murphy, Strachan, Lewis \& Buckley 2016, adapted from Curtis et al, 2009} & $\begin{array}{l}\text { HISTORY: Collection of the presenting } \\
\text { problem and health history. }\end{array}$ \\
\hline & $\begin{array}{l}\text { IDENTIFY RED FLAGS: Historical and } \\
\text { physiological indicators of urgency to } \\
\text { recognise potential and actual serious } \\
\text { illness. Notify red flags to senior } \\
\text { medical officer asap. }\end{array}$ \\
\hline & $\begin{array}{l}\text { ASSESSMENT: Include: primary survey; } \\
\text { A-G, focused head-to-toe; vital signs; } \\
\text { inspection, auscultation, and palpation. }\end{array}$ \\
\hline & $\begin{array}{l}\text { INTERVENTIONS: Should be evidence } \\
\text { based and prioritised based on } \\
\text { assessment. }\end{array}$ \\
\hline & $\begin{array}{l}\text { DIAGNOSTICS: Initiate investigations to } \\
\text { gain clinical picture and inform } \\
\text { treatment decisions. }\end{array}$ \\
\hline \multicolumn{2}{|c|}{$\begin{array}{l}\text { REASSESS: The evaluation of care and monitoring of patient progress. Maintain a structured approach, } \\
\text { repeat at appropriate intervals per condition of the patient. } \\
\text { COMMUNICATE: Verbal/non-verbal skills necessary to effectively communicate with patients, families } \\
\text { and clinicians. Use structured approach for clinical handover; graded assertiveness to escalate if need- } \\
\text { ed; accurate and comprehensive clinical documentation. }\end{array}$} \\
\hline
\end{tabular}

\section{Figure 1}

HIRAID Emergency Nursing Framework 


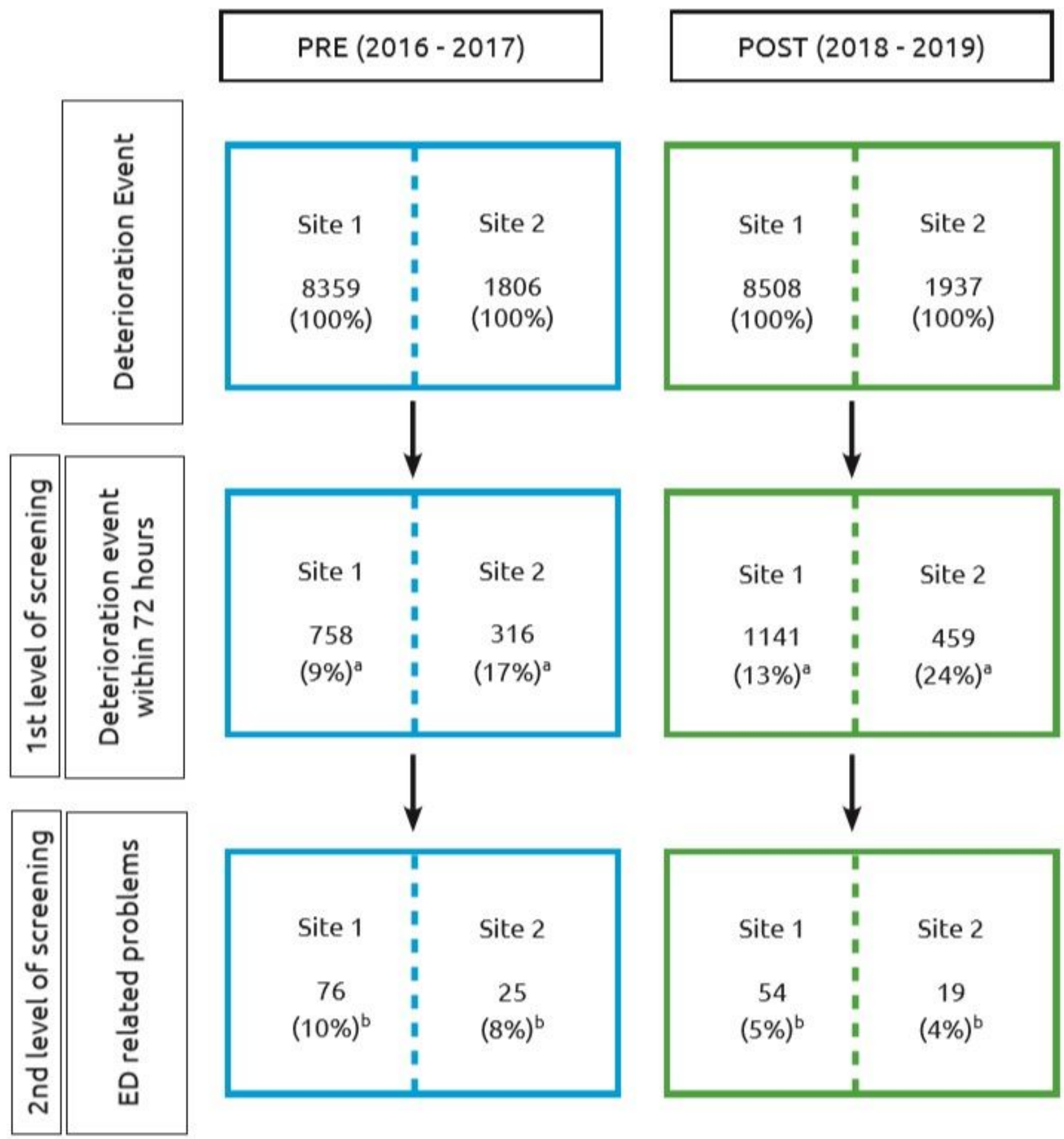

a Percentages calculated by the numbers in the 1st level of screening divided by total deterioration event numbers

${ }^{b}$ Percentages calculated by the numbers in the 2 nd level of screening by the numbers in the 1 st level of screening

Figure 2

HIRAID pre and post cohort case selection and screening

\section{Supplementary Files}

This is a list of supplementary files associated with this preprint. Click to download. 
- HIRAIDISStaRIchecklist20201002kc.docx 